\title{
Editorial
}

\section{Incremental cost-effectiveness of adult spinal deformity surgery}

Christopher I. ShafFrey, M.D., and Justin S. Smith, M.D., Ph.D.

\footnotetext{
Department of Neurosurgery, University of Virginia, Charlottesville, Virginia
}

In the current era of diminishing health care financial resources, an increasing prioritization of treatments and procedures based upon recognized health metrics will occur. Spine surgery is undergoing increasing scrutiny because of a lack of consensus on both the indications for surgery and the specific choices for particular procedures. There is widespread geographic variability in spine surgery rates compared with other surgical procedures, particularly for spinal fusions. ${ }^{2}$ Multiple studies evaluating operative and nonoperative treatments for common spinal disorders have demonstrated substantial variability in decisions regarding nonoperative management, when to operate, who to operate on, when to perform a fusion, and what the appropriate indications are for the various types of spinal implants.

The surgical management of adult spinal deformity (ASD) is rapidly growing despite the high costs and frequent complications associated with these procedures. From 2002 to 2007, the rate of complex fusion procedures in the Medicare population increased 15-fold and was accompanied by a $5.6 \%$ incidence of life-threatening complications and a 30-day readmission rate of $13 \% .^{2,7}$ The growth of these complex deformity procedures is partially driven by the poor ability of nonoperative management of ASD to improve pain and function. A recent study of adult patients with scoliosis treated using nonoperative techniques found that there were no significant improvements in any of the health-related quality of life (HRQOL) outcome measures over the evaluation period. ${ }^{3}$ These patients had substantial nonoperative resource utilization, with the total cost averaging $\$ 14,022$ in the highsymptom patients over the 2 -year study period. ${ }^{3}$

To appropriately assess the value of operative and nonoperative spine care, it is important to select a quality measure that can be applied to cost-effectiveness analysis and compared across other disease states. ${ }^{7}$ The quality-adjusted life year (QALY) is frequently used in cost-effectiveness research, where cost per QALY gained is the unit of measurement to assess the value of health care interventions. ${ }^{7}$ Incremental cost-effectiveness ratios (ICER) compare two or more interventions by looking at the incremental cost and utility of one intervention compared with another intervention, where cost is measured in monetary units and utility is measured by QALYs gained. A recent study estimated the ICERs for decompression and decompression with fusion for focal lumbar spinal stenosis versus total hip arthroplasty and total knee arthroplasty for osteoarthritis and found that the 5-year ICERs were \$21,702/QALY for total hip arthroplasty, \$28,595/QALY for total knee arthroplasty, \$12,271/ QALY for spinal decompression, and \$35,897/QALY for spinal decompression with fusion. ${ }^{6}$ It was concluded that surgical management of focal lumbar spinal stenosis for those in whom medical management has failed results in similar median 5-year and lifetime cost utility compared with those of total hip arthroplasty and total knee arthroplasty. ${ }^{6}$

The paper "Incremental cost-effectiveness of adult spinal deformity surgery: observed quality-adjusted life years with surgery compared with predicted qualityadjusted life years without surgery" by McCarthy et al. attempts to assess the incremental cost-effectiveness of surgical versus nonsurgical treatment of ASD. ${ }^{5}$ Considering the large cost of managing ASD, a comparison of surgical versus nonsurgical treatment using validated HRQOL measures that permit the calculation of ICERs is extremely important. In order to accurately compare costeffectiveness, the patient populations included in the study must accurately reflect the spectrum of patients seeking medical attention for ASD and must also reflect the total costs of operative and nonoperative management. If narrowly selected cohorts of patients are included, it reduces the potential for the study to be widely applied across the ASD population.

There are several aspects of this study that could potentially bias the analysis toward greater cost-effectiveness of surgical treatment of ASD. The incremental effect of surgical treatment relative to nonsurgical treatment in this study was based on a regression model developed using preoperative data from patients who first visited the physician and were followed for a time but ultimately underwent surgical intervention. The resulting model was used to predict hypothetical future QALYs for the operative cohort if they had not undergone surgical treatment. Considering many ASD patients never require surgical intervention, the group of patients included for regression 
analysis in this study likely reflects a smaller subset of deteriorating, high-symptom patients rather than the entire spectrum of ASD patients receiving nonoperative care.

This paper by McCarthy et al. considered only the hospital costs incurred for surgery and did not consider the cost of subsequent nonsurgical treatments in the surgical cohort. Although surgery for ASD clearly has demonstrated HRQOL benefits in selected patients, it is unusual for patients to be symptom free. ${ }^{8}$ Many patients who have undergone surgical management of ASD subsequently require outpatient interventions such as physical therapy and pain medications, costs that were not captured in this study. These treatments could substantially increase the cost for the surgical cohort over a 10-year time frame. This study also does not capture costs related to lost productivity, costs of rehabilitation, or family burden during the prolonged recovery period following ASD surgery.

Results of this study were projected through a 10 year follow-up period. ${ }^{5}$ This study utilized readmission data (23 patients) during the collection period to calculate the QALYs gained. It is highly likely that additional cases of revision surgery will be required beyond the 36-month time period. Revision rates for primary spine fusion procedures for ASD range from $9 \%$ to $45 \%$, with longer length of follow-up associated with greater revision rates. ${ }^{4}$ Bridwell and associates evaluated 113 patients entered into a multicenter database with complete preoperative, 2-year, and 3- to 5-year follow-up data and found that $10 \%$ of patients experienced a new complication at the 3- to 5-year point, most commonly due to implant failure/nonunion and/or junctional kyphosis. The patients who developed these delayed complications had significantly worse clinical outcomes. ${ }^{1}$ Another study evaluated the revision rates for ASD surgical procedures and found 94 of 455 patients underwent further surgical procedures for a revision rate of $21 \% .^{4}$ Thirty-four percent of these revision patients required an additional revision surgery beyond 36 months of follow-up. ${ }^{4}$

The authors should be congratulated in taking the first steps toward evaluating the incremental cost-effectiveness of operative and nonoperative management of ASD. This analysis must be viewed with caution because it likely represents a best-case scenario for the operative management and a worst-case scenario for the nonoperative management of ASD. As better and more detailed cost and outcome data become available, such as those being gathered in the ongoing Multicenter NIH-Sponsored Prospective Study of Quality of Life in Adult Scoliosis (ASLS) study, a more accurate determination of the cost- effectiveness of interventions for ASD can be performed using similar techniques.

(http://thejns.org/doi/abs/10.3171/2014.3.FOCUS14115)

\section{Disclosure}

Dr. Smith serves as a consultant to Biomet, Globus, DePuy, and Medtronic, and has received support for non-study-related clinical or research effort from DePuy/International Spine Study Group. Dr. Shaffrey has served as a consultant to Biomet, Globus, Medtronic, NuVasive, and Stryker, and has received royalties from, and is a patent holder for, Biomet and Medtronic.

\section{References}

1. Bridwell KH, Baldus C, Berven S, Edwards C II, Glassman S, Hamill C, et al: Changes in radiographic and clinical outcomes with primary treatment adult spinal deformity surgeries from two years to three- to five-years follow-up. Spine (Phila Pa 1976) 35:1849-1854, 2010

2. Deyo RA, Mirza SK, Martin BI, Kreuter W, Goodman DC, Jarvik JG: Trends, major medical complications, and charges associated with surgery for lumbar spinal stenosis in older adults. JAMA 303:1259-1265, 2010

3. Glassman SD, Carreon LY, Shaffrey CI, Polly DW, Ondra SL, Berven SH, et al: The costs and benefits of nonoperative management for adult scoliosis. Spine (Phila Pa 1976) 35: $578-582,2010$

4. Kelly MP, Lenke LG, Bridwell KH, Agarwal R, Godzik J, Koester L: Fate of the adult revision spinal deformity patient: a single institution experience. Spine (Phila Pa 1976) 38: E1196-E1200, 2013

5. McCarthy I, O'Brien M, Ames C, Robinson C, Errico T, Polly DW Jr, et al: Incremental cost-effectiveness of adult spinal deformity surgery: observed quality-adjusted life years with surgery compared with predicted quality-adjusted life years without surgery. Neurosurg Focus 36(5):E3, 2014

6. Rampersaud YR, Tso P, Walker KR, Lewis SJ, Davey JR, Mahomed NN, et al: Comparative outcomes and cost-utility following surgical treatment of focal lumbar spinal stenosis compared with osteoarthritis of the hip or knee: part 2-estimated lifetime incremental cost-utility ratios. Spine J 14:244-254, 2014

7. Rihn JA, Currier BL, Phillips FM, Glassman SD, Albert TJ: Defining the value of spine care. J Am Acad Orthop Surg 21:419-426, 2013

8. Smith JS, Shaffrey CI, Glassman SD, Berven SH, Schwab FJ, Hamill CL, et al: Risk-benefit assessment of surgery for adult scoliosis: an analysis based on patient age. Spine (Phila Pa 1976) 36:817-824, 2011

Please include this information when citing this paper: DOI: 10.3171/2014.3.FOCUS14115 